\title{
Importance of Metal-Adsorbate Interactions for the Surface-enhanced Raman Scattering of Molecules Adsorbed on Plasmonic Nanoparticles
}

\author{
L. Guerrini • Z. Jurasekova • C. Domingo • \\ M. Pérez-Méndez • P. Leyton - M. Campos-Vallette • \\ J. V. Garcia-Ramos • S. Sanchez-Cortes
}

Received: 30 April 2007 / Accepted: 22 August 2007 /Published online: 12 September 2007

(C) Springer Science + Business Media, LLC 2007

\begin{abstract}
The interaction between adsorbates of different nature and plasmonic nanoparticles is reviewed here on the basis of the work done in our laboratory in the past few years. The paper is structured for analyzing the interaction of adsorbates with metal nanoparticles as function of the interacting atom $(\mathrm{O}, \mathrm{N}$, or $\mathrm{S})$ and the adsorbate conformation. In the study of the adsorption of molecular species on metals, it is necessary to take into account that different interaction mechanisms are possible, leading to the existence of different molecular forms (isomers or conformers). These forms can be evidenced by changing the excitation wavelength, due to a resonant selection of these wavelengths. Charge-transfer complexes and electrostatic interactions are the usual driving forces involved in the interaction of adsorbates on metal surfaces when these metallic systems are used in wet conditions. The understanding of the metal-adsorbate interaction is crucial in the surface functionalization of metal surfaces, which has a growing importance in the development of sensing systems
\end{abstract}

L. Guerrini $\cdot$ Z. Jurasekova $\cdot$ C. Domingo $\cdot$

J.V. Garcia-Ramos · S. Sanchez-Cortes $(\square)$

Instituto de Estructura de la Materia, CSIC,

Serrano, 121, 28006 Madrid, Spain

e-mail: imts158@iem.cfmac.csic.es

M. Pérez-Méndez

Instituto de Ciencia y Tecnología de Polímeros, CSIC,

Juan de la Cierva, 3, 28006 Madrid, Spain

P. Leyton

Departamento de Química, Facultad de Ciencias Básicas,

Universidad de Antofagasta, Casilla, 170 Antofagasta, Chile

M. Campos-Vallette

Faculty of Sciences, University of Chile,

PO Box 653, Santiago, Chile or optoelectronic devices. In relation to this, special attention is paid in this work to the study of the adsorption of calixarene host molecules on plasmonic nanoparticles.

Keywords SERS · Plasmonic nanoparticles · Adsorption . Metal-molecule interaction

\section{Introduction}

The optoelectronic properties of metallic nanostructures have a remarkable technological importance $[1,2]$. These properties are determined by surface plasmon resonances, which are strongly dependent on particle size and shape. For this reason, these particles have been lately called plasmonic nanoparticles (NPs) [3].

Resonant excitation of plasmons can lead to large local enhancements of the incident electromagnetic field at the NP surface, resulting in dramatically large enhancements of the cross section for optical spectroscopies such as surfaceenhanced Raman scattering (SERS).

SERS is nowadays a useful analytical technique that has been extensively employed in the identification and most probable orientation of molecules adsorbed onto a surface. The high sensitivity of this technique is mainly due to the giant electromagnetic enhancement taking place on these nanostructured metal surfaces [4] (electromagnetic mechanism, EM). However, a part of the total enhancement is also attributed to a resonant effect associated to an adsorbatemetal charge transfer (CT) [5], leading to the so-called CT mechanism for SERS.

The optical properties of metal substrates are not the only limiting factor in SERS. The chemical structure of the studied adsorbate is also important to ensure a strong SERS 
signal. In fact, a certain degree of interaction between the adsorbate and the metal surface is required to have intensification of the Raman signal through the mechanisms involved in SERS enhancement. The EM mechanism needs the adsorbate to be close enough to the surface because of the short range of the surface-enhanced electromagnetic field $[5,6]$. On the other side, the CT mechanism implies an effective metal-adsorbate interaction for the $\mathrm{CT}$ process to take place [7].

Although the establishment of a metal-adsorbate interaction is an important condition in surface-enhanced optical techniques, we consider that a rather poor amount of attention has been devoted so far to this important issue under a general point of view. This kind of interaction is not only important for the optical enhancement but also for the functionalization of metal NPs leading to supramolecular assemblies of metal and advanced organic or biomolecular species, which are interesting in sensing, electronic, and solar cell devices [8].

In this work we present a review of the metal-adsorbate interactions studied in our recent and past experience when applying the SERS technique to study the adsorption of molecules with different chemical structures. Our experience is mainly based on the adsorption of molecules in wet systems where a diffusion of the adsorbate from the bulk to the metal surface is needed. In these systems, the presence of oxygen, nitrogen, and sulfur atoms in the adsorbate is crucial for a strong metal-adsorbate interaction to occur. Thus, we have decided to classify the studied molecules by taking into account the presence of these atoms in the molecule.

Indeed, the affinity of adsorbates toward the metal surface is highly affected by the nature of the metal interface. In the case of metal nanostructures, the interface is negatively charged, the negative potential or zeta potential value depending on the method employed to prepare the nanostructures [9]. This means that positively charged adsorbates will present the most enhanced vibrational spectra due to their high preference to adsorb onto the surface. In wet systems, the high polar nature of the interface makes the adsorption of highly apolar molecules such as polycyclic aromatic hydrocarbons difficult. However, the affinity of the adsorbate towards the metal can be increased by modifying the chemistry of the interface. This can be done by displacing the anions adsorbed onto the metal surface, e.g., by using halide anions in the case of Ag, which show a strong affinity for this metal [10].

Another strategy to approach nonactive molecules to the surface is a proper functionalization of metal NPs. In the past few years, we have also paid much attention to the modification of surface metal NPs through the adsorption of molecules displaying a double functionalization, i.e., a high affinity to the metal and to the ligand [11]. These species cannot only modify the chemical properties of the interfaces but also organize the metal NPs into controlled architectures with more advanced physico-chemical properties.

SERS spectra contain valuable structural information, which serves to help us understand the interaction mechanism of the corresponding adsorbate on the metal surface. The interaction of molecular groups with the metal induces changes in the position and intensity of vibrational bands. This fact allows the interpretation of the interaction mechanism occurring between adsorbed molecules and the metallic surface. These changes mainly affect those groups directly involved in this interaction. In addition, the interaction with the metal leads to the formation of a new metal-molecule bond, which is usually recognized in the low wavenumber region of the Raman spectrum [12]. However, this region is difficult to analyze because of the large amount of different species that may be adsorbed onto the surface. The orientation adopted by the adsorbed species can be inferred from the spectra as well, using the helpful selection rules provided by SERS effect [13-15]. According to them, which in many occasions have been also named propensity rules, the vibrational modes perpendicularly oriented with respect to the metal surface will undergo the maximum SERS intensification. Another reason to have a selective intensification of certain modes is the resonance Raman effect, which may occur when using excitation wavelengths falling in the adsorbate absorption region.

The mini review presented here does not intend to be a general study of all the molecules studied so far by means of SERS. Our purpose is to summarize the experience carried out in our group for a wide spectrum of adsorbates of different natures.

\section{Experimental details}

\section{Preparation of the metal NPs}

There is a large variety of SERS substrates, including electrodes, metal island films, and NPs in suspension or colloids. However, wet chemistry provides an inexpensive and versatile approach to metal NP fabrication [16]. Colloidal NPs are commonly used for SERS studies in suspension as sols, or the analyte-colloid system is cast onto a sustaining surface and allowed to dry [9]. The use of colloidal NPs in SERS measurements has several advantages because they minimize the danger of burning the sample, allowing the use of higher power at the sample and more energetic laser lines. The use of colloidal dispersions permits the acquisition of an average spectrum due to the Brownian motion that governs the colloidal dispersions [17], although a 
diffusion of the analyte to the surface is required. On the other hand, the need of a molecular diffusion ensures the adsorption of only those species that are strongly attached on the surface. The preparation of such systems is mainly accomplished by chemical reduction, although we have also employed other techniques such as ablation, radiolysis, photoreduction [18], and pulsed-laser deposition [19]. The addition of the corresponding adsorbate can be made by solving it in a proper solvent and adding an aliquot to the NPs in suspension or cast to the metallic films, depending on the cases.

\section{Instrumentation}

FT-Raman spectra of the solid compounds placed in a brass holder were obtained with a Bruker RFS 100/S instrument by using the line at $1,064 \mathrm{~nm}$, provided by a Nd/YAG laser and a Ge detector cooled by liquid nitrogen. The resolution was set to $4 \mathrm{~cm}^{-1}$ and the $180^{\circ}$ geometry was employed. The output laser power was $50 \mathrm{~mW}$. Up to 1,000 scans were accumulated.

SERS spectra at 785, 632.9, and $514.5 \mathrm{~nm}$ were measured with a Renishaw Confocal Raman Microscope System RM2000 equipped with diode, $\mathrm{He}-\mathrm{Ne}$, and $\mathrm{Ar}+$ lasers, respectively, a Leica microscope, and an electrically refrigerated CCD camera. The laser power in the sample was $2.0 \mathrm{~mW}$. This system allows mapping and imaging of the sample.

\section{Results and discussion \\ O-containing adsorbates}

SERS has been reported for many O-containing adsorbates (OCA), including water [20]. However, the most intense SERS signals were obtained for OCA compounds bearing $\pi$-electron systems. Carboxylate and ester groups can interact strongly with $\mathrm{Ag}$ or $\mathrm{Au}$ through $\mathrm{CT}$ coordination bonds involving the empty orbitals of metals. The interaction of citrate with silver and gold has been extensively studied by SERS [21], being of marked importance due to the universal employ of citrate-based Ag NPs to prepare metal substrates for SERS. This interaction can be stronger when the carboxylate group is attached to an aromatic system in benzoic acids. In the case of alcohols, whereas alkylic alcohols are almost inactive in SERS, the aromatic ones, i.e., phenols, seem to strongly interact with metals. In some cases, this interaction is so strong that a chemical degradation occurs in the adsorbate. This is the case of polyphenols with $\mathrm{OH}$ groups in ortho-position, such as catechol [22], caffeic acid [23], or gallic acid [24]. When a chemical modification in the adsorbate is induced upon adsorption on the metal, a completely different SERS spectrum is recorded. This is illustrated in Fig. 1, where the SERS spectrum of adsorbed cathecol is shown (Fig. 1b) together with the Raman spectrum of the molecule in solid state (Fig. 1a).

Alkyl ketones are also inactive in SERS due to their low affinity regarding the metal surfaces. On the contrary, aromatic quinones display a stronger affinity with respect to the metal surface, which can lead to a chemical reduction in the corresponding hydroquinone species. The chemical stability of quinones can be preserved on the metal surface if the keto is protected by adjacent phenolic groups. This is the case, for example, of phenolic naphtoquinones and anthraquinones. In previous works we have extensively

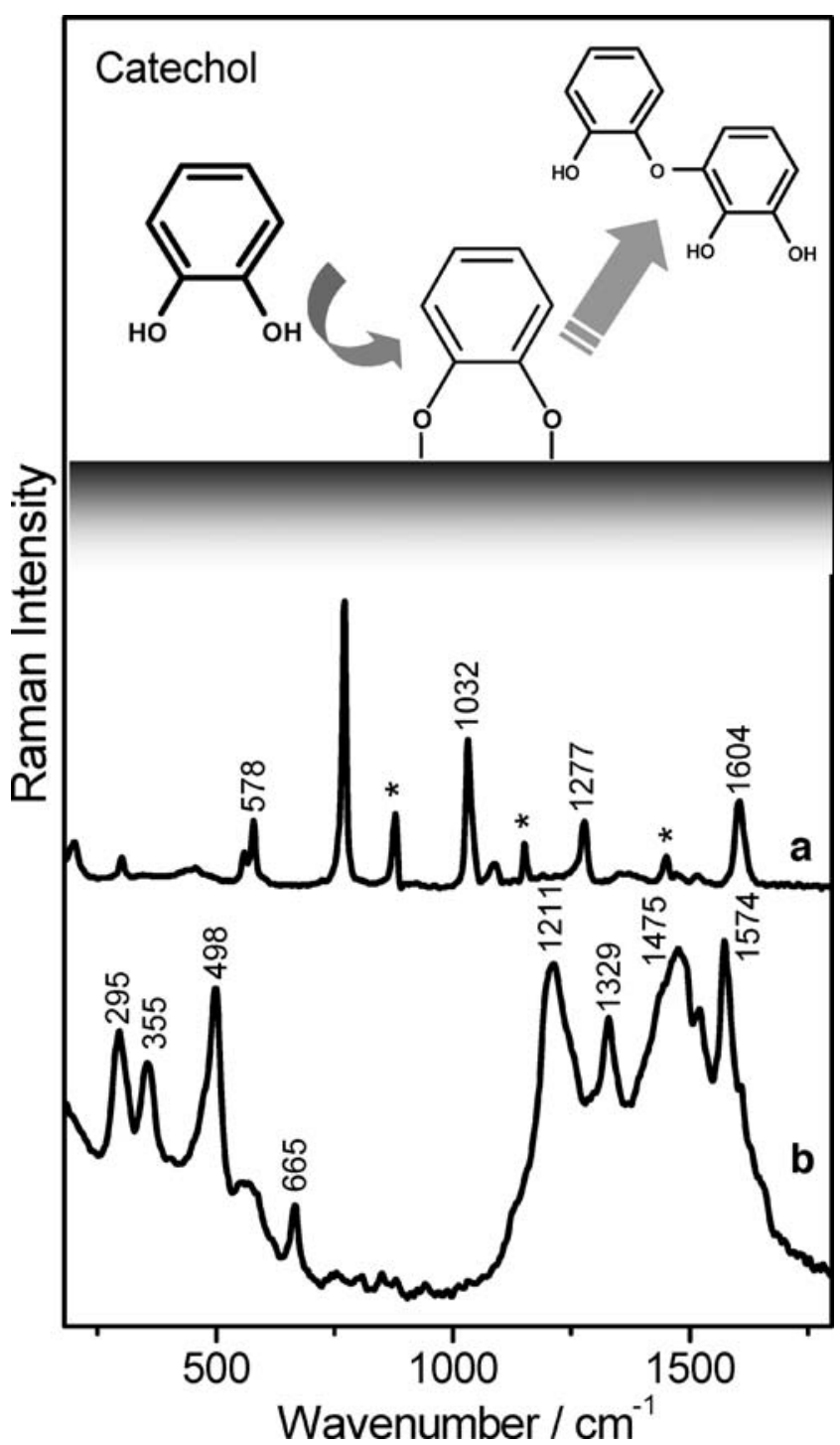

Fig. 1 Top: Scheme representing the adsorption of cathecol on $\mathrm{Ag}$ NPs by the o-diphenol moiety and the subsequent polymerization resulting from this adsorption. Bottom: Raman spectra of solid cathecol (a) and cathecol adsorbed onto Ag NPs showing the spectral changes related to this polymerization 
studied these molecules, concluding that a chelate coordination complex of $-\mathrm{OH}$ and $\mathrm{C}=\mathrm{O}$ groups and the metal is formed $[25,26]$. This interaction is favored at high $\mathrm{pH}$ due to ionization of the phenol group. The study of these molecules on Ag NPs has revealed that different molecular forms are possible on the metal. This is shown in Fig. 2 for naphtazarin. The differently adsorbed molecules can be detected by marker vibrational bands. Even if a molecular species is at very low concentration, its SERS spectrum can be manifested when resonant conditions are taking place. This is why the SERS spectrum of a molecule can dramatically change on varying the excitation wavelength. In the case of naphtazarin, a few molecules are adsorbed under the neutral form on the Ag surface at neutral $\mathrm{pH}$. These neutral molecules are manifested in the SERS when moving the excitation from 1,064 to $514.5 \mathrm{~nm}$ (Fig. 2).

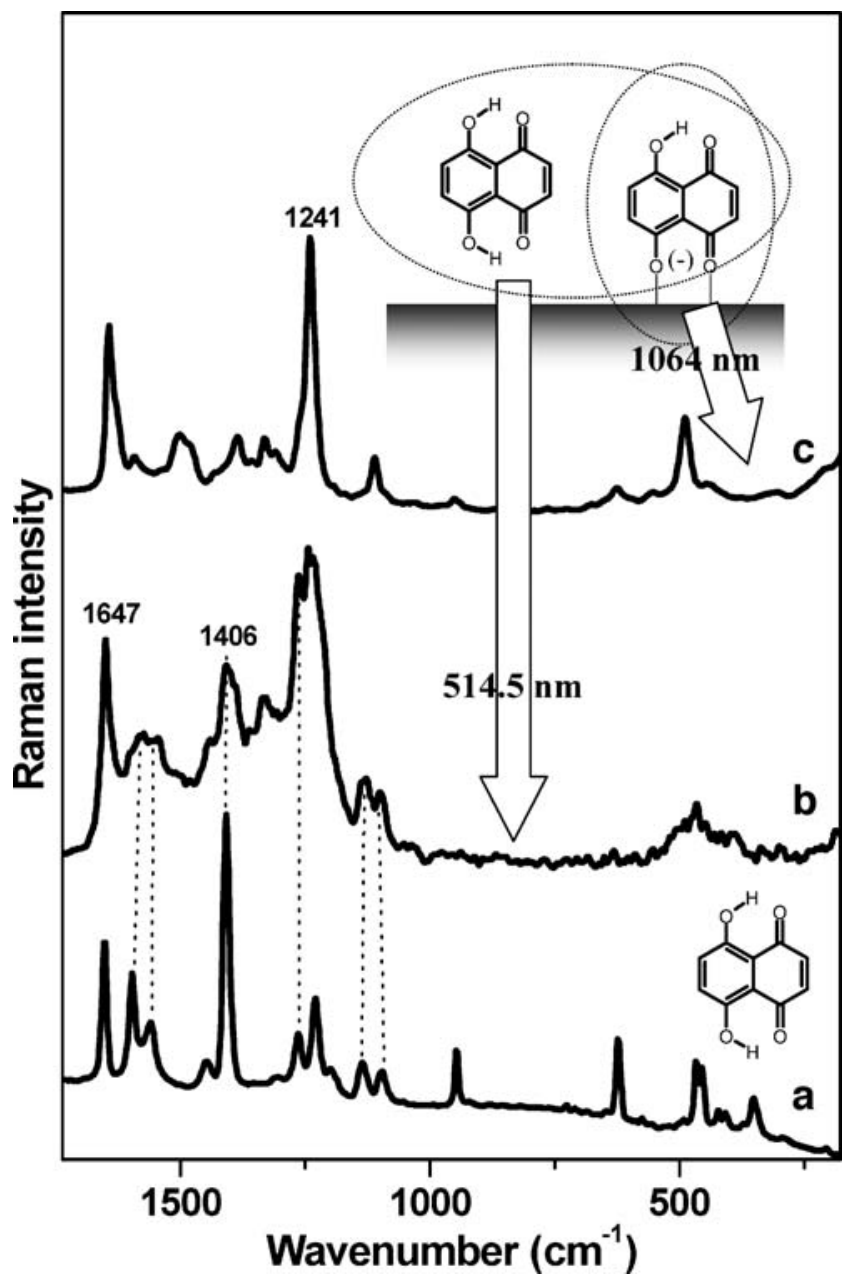

Fig. 2 Raman spectrum of solid naphtazarin (a) and SERS spectrum of naphtazarin $\left(10^{-5} \mathrm{M}\right)$ on Ag NPs by exciting at 514.5 (b) and $1,064 \mathrm{~nm}(\mathbf{c})$. At $1,064 \mathrm{~nm}$, the spectrum is dominated by the ionized adsorbate form resulting from the interaction with the metal (top scheme), which is the dominant species on the surface. However, the still presence of neutral molecules on the metal is evidenced when exciting at 514 (see bands marked with dotted lines), suggesting the formation of multilayers on the surface
Flavonoids are a class of OCA compounds where many $\mathrm{O}$ atoms coexist in different aromatic moieties. In these compounds, cathecol and benzopyrone moieties can exist together in the same molecule. The study of the adsorption of these molecules on metal surfaces is interesting to compare with the metallic complexes in solution. On the other hand, the interaction with metals is part of the biological activity of these biomolecules, and it is related to their antioxidant activity. The SERS spectra of flavonoids indicate that there are two main instability sources in these molecules: the cathecol ring and benzopyrone [27]. The adsorption of flavonoids on metal surface can induce a chemical change similar to that found for cathecol.

The adsorption of OCA is markedly influenced by the electric potential of the metal surface, which in turns depends on the NPs' preparation method. Because citrate NPs are highly negative due to the adsorbed citrate anions on the surface, negatively charged adsorbates have a lower affinity for this surface. On citrate NPs, the affinity is highly affected by the $\mathrm{pH}$ due to the influence of $\mathrm{pH}$ on the protonation state of citrate. The affinity of carboxylate adsorbates for this surface is strongly enhanced when lowering the $\mathrm{pH}$ because of the negative charge reduction of the adsorbate.

Halide ions markedly affect the metal-adsorbate interaction, mainly in the case of Ag, due to the strong interaction occurring between this metal and halides. In the presence of chloride, many carboxylate species can be removed from the surface, as it occurs in the case of citrate. Chloride also passivates the silver surface so that the catalytic action on O-diphenols is inhibited [28].

$\mathrm{N}$-containing adsorbates

The interaction of N-containing adsorbates (NCA) and metal NPs is usually strong in the case of the aromatic com pounds containing heterocyclic $\mathrm{N}$ atoms. A coordination complex of NCA with metal is possible through a CT between the free electron pair existing in $\mathrm{N}$ atoms and empty orbitals of transition metals. N-containing heterocycles are among the most studied molecules in SERS. In fact, pyridine has been used as a reference adsorbate from the very beginning of the SERS effect [29]. Many other SERS works have been published on the interaction of other heterocycle NCA, such as bypyridine [30], pyrazine [31], etc.

The interaction of amine compounds with metal NPs is, in general, strong. Electrostatic forces seem to play an important role in the interaction with the negatively charged surface of metal NPs, due to the positive charge of amines at neutral $\mathrm{pH}$, and seem to take place through the formation of an ionic pair with the anion species adsorbed on the metal surface. The study of the adsorption of aliphatic 
amines in SERS has been carried out mainly in the case of aminoacids or peptides [32, 33], for which a preferential interaction through the N-terminal moiety of the oligopeptides was seen, thus indicating that the amino interaction with the metal is preferred to the interaction through the carboxylate one. Aromatic amines display a lower basicity; hence, their interaction with metals through electrostatic forces is weaker. Amines are strongly attached to the surface at protonated state, i.e., when they are positively charged. This is why their interaction strength decreases at alkaline $\mathrm{pH}$.

Acridine derivatives are a special case of $\mathrm{N}$-containing heterocyclic compounds that strongly interact with metal surfaces through the formation of a coordination bond with the metal. However, on lowering the $\mathrm{pH}$, they form an ionic pair through the protonated acridine $[34,35]$. When two $\mathrm{N}$ atoms of different nature exist in acridine derivatives, the interaction with the metal can take place through different mechanisms, leading to the coexistence of adsorbates with a different structure. This is the case of 9-aminoacridine (Fig. 3), which can interact with Ag through the amino form or through the corresponding imino form in a stronger interaction [36]. The imino form is favored at low $\mathrm{pH}$ and in the presence of chloride, as it may imply the formation of an ionic pair with the halide. The coexistence of different adsorbates is also the reason why different SERS spectra are recorded when varying the excitation wavelength or the adsorbate concentration (Fig. 3).

The study of the interaction of amide-containing molecules and metals has been mainly restricted to biological

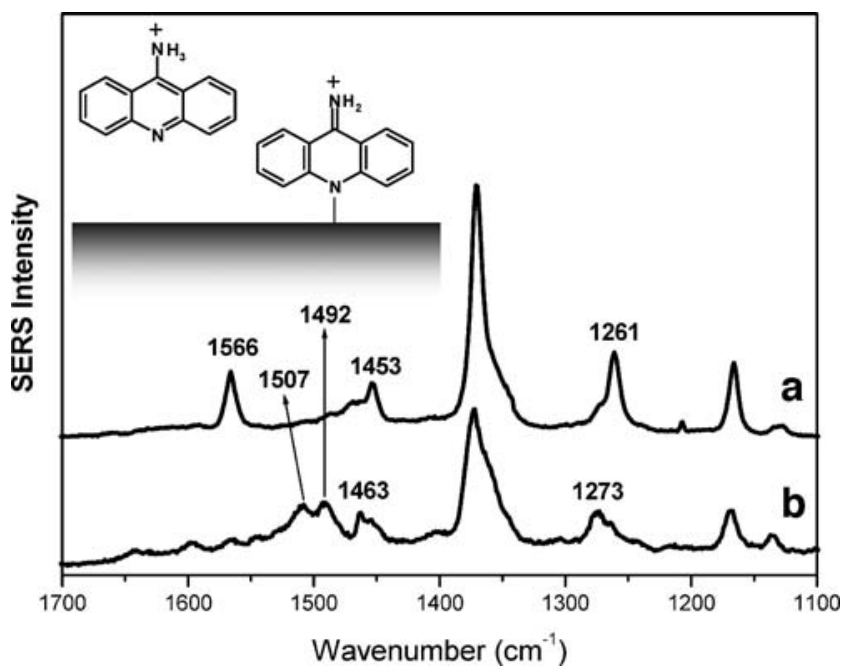

Fig. 3 SERS spectra of 9-aminoacridine on Ag NPs Raman spectrum at the two extreme conditions at which this molecule shows completely different spectral profiles corresponding to the amino and imino forms (see top scheme): at $5 \times 10^{-5} \mathrm{M}$ and in the absence of chloride (a), where the spectrum of the amino form is seen, and at $5 \times 10^{-3} \mathrm{M}$ and in the presence of chloride $\left(3 \times 10^{-2} \mathrm{M}\right)(\mathbf{b})$, where the characteristic bands of the imino form emerges in the SERS spectrum. Excitation at $514.5 \mathrm{~nm}$

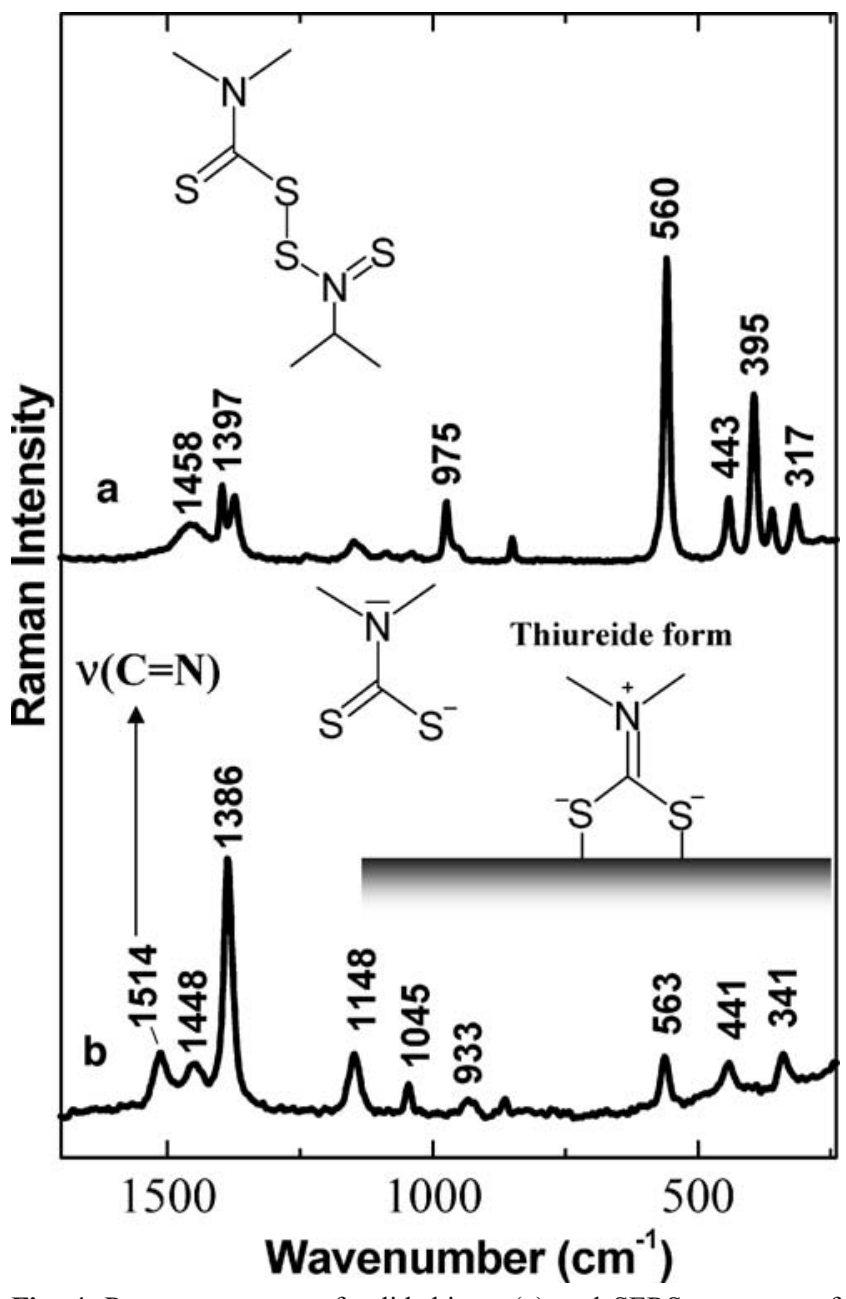

Fig. 4 Raman spectrum of solid thiram (a) and SERS spectrum of thiram $\left(10^{-5} \mathrm{M}\right)$ on $\mathrm{Ag}$ NPs by exciting at $514.5 \mathrm{~nm}$ (b) showing the bands related to the thioureide form induced upon adsorption on the metal surface

compounds bearing this group in their structure. This is the case of peptides and nucleic bases and their derivatives. Nucleic bases display a high affinity to be adsorbed on metal surfaces due to the presence of a large number of heterocyclic $\mathrm{N}$ atoms able to form strong coordination bonds with metals. The interaction of these compounds with the metal is verified by means of heterocyclic $\mathrm{N}$ atoms and $\mathrm{C}=\mathrm{O}$ groups in the case of amidic molecules. For instance, cytosine and its methyl derivatives may interact with metal surfaces through different mechanisms depending on the relative position of the methyl groups. The interaction of cytosine with $\mathrm{Ag}$ colloids leads to an isomerization change from amino-keto to imino-enolic forms due to a cascade effect from the amino group in position 4 to the amide moiety in positions 1 and 2 [37-39]. The formation of an imino structure in position 4 is highly hindered if a methyl group is introduced in position 5, i.e., in 5-methylcytosine and 1,5-dimethylcytosine. In the latter case, only an enolization of positions 1 and 2 is induced. 


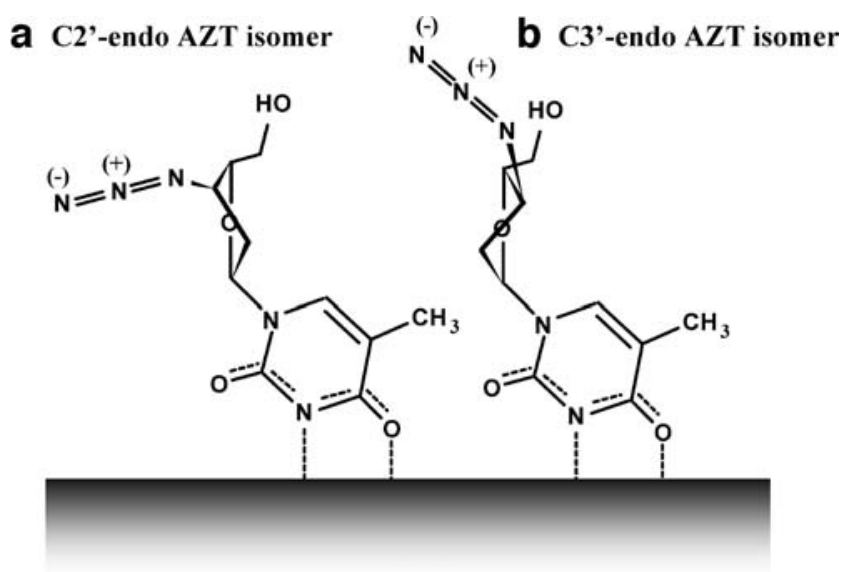

Fig. $5 \mathrm{C} 2$ '-endo to $\mathrm{C} 3$ '-endo isomerization deduced from the SERS spectra of AZT because of the molecular rearrangement on the metal surface

The different adsorptions of 5-methyled cytosine derivatives are also related to the different electric behavior demonstrated by methylated cytosine with important consequences in DNA base pairing.

An interesting case of metal-adsorbate interaction is the adsorption of bypyridinium dications on $\mathrm{Ag}$ and $\mathrm{Au}$. These compounds are able to interact with metal surfaces through the formation of CT complexes with the anions adsorbed onto the metal surface. The adsorption of methyl-viologen on metallic surfaces has already been studied on electrodes due to the special electrochemical behavior of these molecules [40]. This interaction is so strong in the case of the bis-acridinium dye lucigenin that a structural change in the environment of $\mathrm{N}$ atoms occurs [41, 42].

Nitro compounds are also capable of interacting with the metal surface due to the electron donor ability of this group. In fact, the introduction of a nitro group in molecules that are not able to interact with metal surfaces, such as polycyclic aromatic hrdrocarbons (PAHs), induces their adsorption through the $\mathrm{O}$ atoms [43].

\section{S-containing adsorbates}

S-containing adsorbates usually present the highest affinity to be adsorbed on Ag or Au surfaces. In fact, thiols are widely used in surface functionalization on these metals $[44,45]$. The formation of a complex with the metal usually implies the breakdown of the $-\mathrm{S}-\mathrm{H}$ bond and the formation of a new metal-S bond.

We have seen that this interaction could be stronger in the case of dithiocarbamate (DTC) compounds (Fig. 4) because a $\mathrm{CT}$ from the $\mathrm{N}$ atom to the metal is induced leading to the formation of a thioureide compound [46, 47], characterized by the $v(\mathrm{C}=\mathrm{N})$ band at $1,514 \mathrm{~cm}^{-1}$ (Fig. $4 \mathrm{~b}$ ). Monodentate and bidentate adsorptions of DTC molecules give rise to characteristic $\mathrm{C}-\mathrm{S}$ and $\mathrm{C}=\mathrm{S}$ bands, which change depending on the surface coverage.

\section{Adsorption of large molecules on the metal surface}

Large molecules are more difficult to study in SERS, not only because of the higher structural complexity but also due to the lower tendency toward adsorption and the longer distance of SERS-active groups to the surface. Few works have been reported so far on the SERS of proteins, although many authors have largely demonstrated that proteins can be readily adsorbed onto metal surfaces. Nucleic acids can readily interact with the metal through the nucleic bases but only if single strands are used. In double-strand DNA, the bases are more protected and, hence, the SERS signal is much weaker. The adsorption and interaction with metal surfaces of nucleotides is controlled by the electrostatic forces derived from the overall charge given by phosphate groups. This is due to the equilibrium established between adsorbed molecules and hydrated molecules in the aqueous bulk medium [48]. The adsorption of these molecules onto the metal surface can induce a structural change in the adsorbate due to the possible deprotonation of $\mathrm{NH}$ groups and the spatial rearrangement of the molecule when selfassembled on the surface.

To illustrate this effect, Fig. 5 shows the conformation analysis of 3'-azido-3'-deoxythumidine (AZT) on Ag NPs [49]. In solution, and at acidic or neutral $\mathrm{pH}$, a mixture of the $C 2^{\prime}$-endo and $C 3^{\prime}$-endo AZT forms coexists, as the existence of bands attributed to both conformations reveals: $1,376,1,203,747$, and $667 \mathrm{~cm}^{-1}$, assigned to the $C 2^{\prime}$-endo
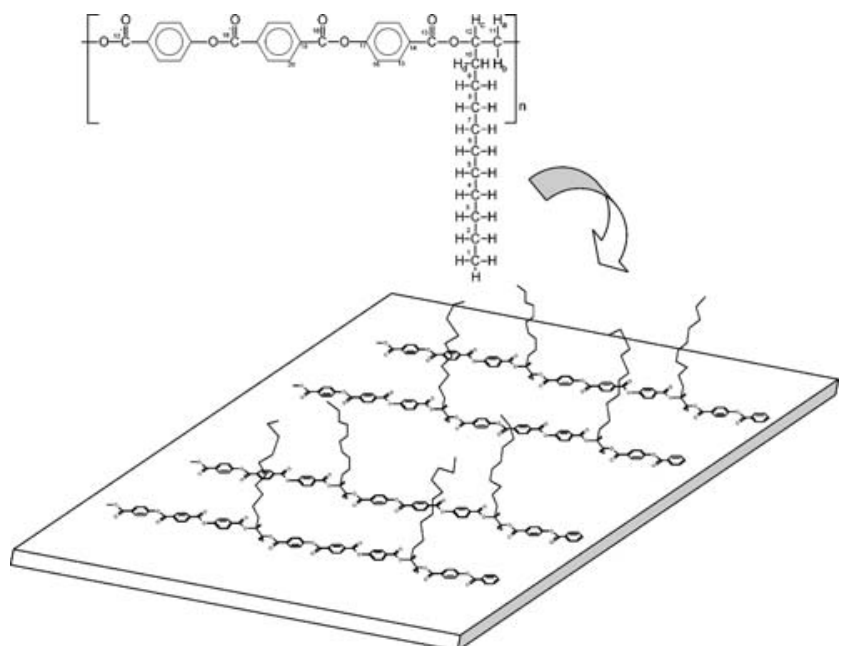

Fig. 6 Adsorption of PTOBDME on Ag NPs displaying the mechanism deduced from the SERS spectra: the terphtalate moiety containing the ester groups directly interacting with the metal, whereas the aliphatic chains are oriented outwards in a disorder structure 


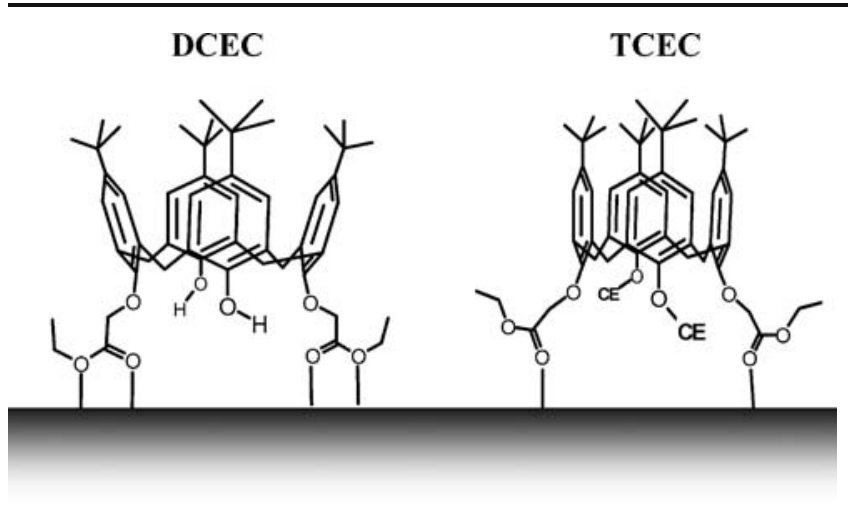

Fig. 7 Adsorption of DCEC and TCEC on Ag NPs showing the bidentate and monodentate interaction mechanism induced by the steric hindrance of the four carboethoxy (CE) groups in the lower rim of TCEC. This hindrance induced the closening on the tert-butyl groups in the upper part of the calixarene, which limits the use of TCEC in PAHs sensing by SERS

conformation, and $1,242,781$, and $642 \mathrm{~cm}^{-1}$, assigned to the C3'-endo one [50]. The adsorption of AZT on Ag induces a deprotonation of $\mathrm{N}(3)$ atom, leading to a conformational change from $C 2^{\prime}$-endo to $C 3^{\prime}$-endo.

Synthetic polymers containing functional groups that tend to interact with metals also display a relative affinity towards the metal surface. Thiophene-containing and pyrrole-containing polymers have been extensively studied by numerous authors [51, 52]. In addition, the estercontaining polymers are also able to interact with metals forming coordination bonds.
We present here two examples concerning the adsorption of ester-containing polymers on metal surfaces: the thermotropic liquid crystal poly [oxy (1,2 - dodecane) oxycarbonyl 1,4 - phenyleneoxycarbonyl - 1,4 - phenylenecarbonyloxy - 1, 4 - phenylenecarbonyl] (PTOBDME), $\left[\mathrm{C}_{34} \mathrm{H}_{36} \mathrm{O}_{8}\right]_{\mathrm{n}}$, Fig. 6, and ester-functionalized calix[4]arenes. PTOBDME shows a high affinity to be adsorbed and self-assembled on $\mathrm{Ag}$ surfaces despite its very low solubility in aqueous media. The adsorption of the polymer involves a deep conformational change of the main chain, consisting in an opening of the helicoidal structure of PTOBDME in suspension, to an extended lineal polymer structure when the polymer is adsorbed on the metal surface [53] (Fig. 6). This structural change may be favored by an interaction of the metal with the aromatic rings via $\pi$-electronic interactions, leading to a small torsional angle between them. Another important structural consequence of this kind of interaction is the order decrease of the aliphatic side chain. The rather parallel orientation of benzene rings with respect to the surface is suggested by the weakness of the $\mathrm{C}-\mathrm{H}$ stretching band and the enhancement of the out-of-plane ring vibrations, appearing below $1,000 \mathrm{~cm}^{-1}$.

\section{Comparative adsorption of functionalized calix[4]arenes}

The diester functionalized calix[4]arene 25,27-dicarboethoxy-26,28-dihydroxy-p-t-butylcalix[4]arene (DCEC) (Fig. 7) also interacts strongly with the surface through
Fig. 8 SERS of DTCX on Ag NPs in absence (bottom) and the presence of pyrene (top). The $\mathrm{C}=\mathrm{S}$ and $\mathrm{C}-\mathrm{S}$ stretching bands inversion are interpreted as due to a relative mono- to bidentate adsorption of DTCX induced by the interaction with pyrene (see schemes on the right). The presence of pyrene bands (upper spectrum) indicates the presence of this pollutant in the complex. The different behavior of DTCX as compared to DCEC is attributed to the steric differences imposed by the lower chains

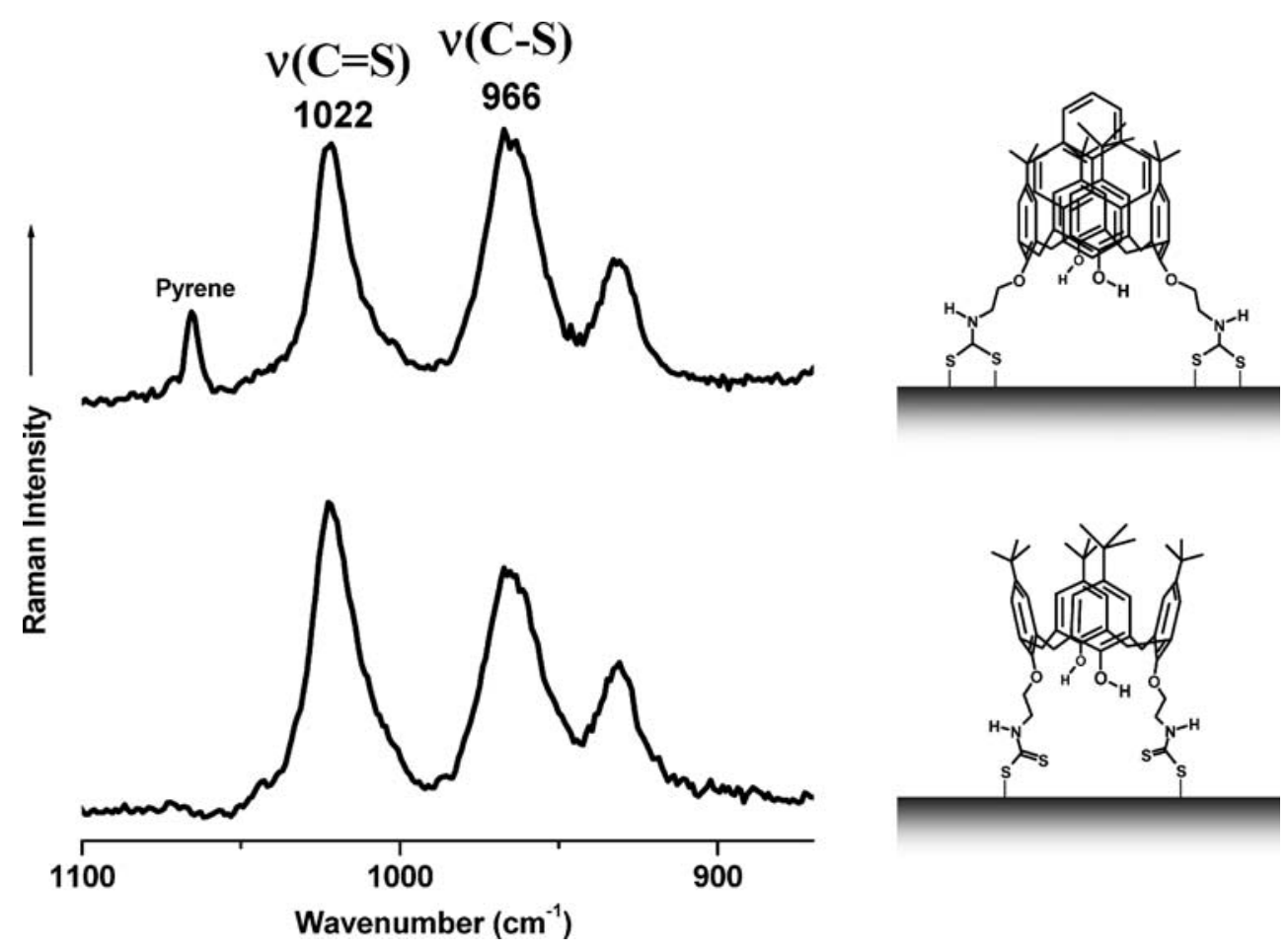


the ester groups. This is deduced from the changes observed for the ester-stretching band, which shifts from 1,759 to $1,749 \mathrm{~cm}^{-1}$ upon adsorption on the surface. The interaction with the metal leads to the formation of a CT complex, which seems to have its absorption band towards $500-600 \mathrm{~nm}$, as deduced from the fact that the SERS spectrum is markedly intensified when exciting at $514.5 \mathrm{~nm}$, whereas very weak signals are obtained at $1,064 \mathrm{~nm}$ [54]. However, SERS spectra provide information of the aromatic groups mainly. The application of surface-enhanced infrared absorption (SEIRA) was very useful in this case to understand the precise adsorption mechanism of DCEC and the corresponding tetra-ester calix[4]arene compound 25,26,27,28-tetracarboetoxy-ptert-butylcalix[4]arene (TCEC) [55], due to the stronger intensity of the groups through which the interaction is verified: the ester ones. The analysis of ester $\mathrm{C}=\mathrm{O}$ bands and the bands corresponding to the carboethoxy moiety [$\mathrm{O}-\mathrm{CH} 2-\mathrm{C}(\mathrm{O})-\mathrm{O}-\mathrm{CH} 2-\mathrm{CH} 3]$, especially those falling in the $1,300-1,000 \mathrm{~cm}^{-1}$, reveal that DCEC is adsorbed with the ester groups forming a bidentate complex with the metal, whereas TCEC forms a monodentate complex (Fig. 7) due to the steric hindrance of the four carboethoxy groups in the lower calixarene rim.

Interestingly, the corresponding amino-functionalized calix[4]arene derivative seems to interact very weakly with $\mathrm{Ag}$ NPs, as deduced from the weak SERS spectrum [56]. Only on $\mathrm{Au}$ NPs was a significant signal obtained. However, the introduction of DTC groups in this amino derivative by means of reaction with $\mathrm{CS}_{2}$ leads to compounds, which are strongly attached on $\mathrm{Ag}$ thanks to the strong interaction of DTC group with metals.

The SERS spectrum of the corresponding DTC calyx[4] arene (DTCX, Fig. 8) shows a band at $198 \mathrm{~cm}^{-1}$, attributed to the $\mathrm{Ag}-\mathrm{S}$ stretching motion [56], indicating that the interaction of DTCX occurs through the DTC group. A consequence of the strong interaction of DTCX with $\mathrm{Ag}$ is the band at $1,514 \mathrm{~cm}^{-1}$, attributed to the partial $\mathrm{C}-\mathrm{N}$ double bond induced by the transformation of DTC group (Fig. 8) into the corresponding thioureide form, due to the lone pair electrons attraction of the nitrogen by the complexation with the metal. The DTCX SERS spectrum is dominated by DTC bands seen at $1,022 \mathrm{~cm}^{-1}$ and $966 \mathrm{~cm}^{-1}$ that have been ascribed to the $v(\mathrm{C}=\mathrm{S})$ and to $v(\mathrm{C}-\mathrm{S})$ motions, respectively, which are characteristic of a thioureide monodentate complex with the metal surface [56]. These bands undergo a shift and subsequent broadening in the presence of Ag NPs as compared to the corresponding band appearing at 1,027 and $963 \mathrm{~cm}^{-1}$ in the Raman of the solid DTCX.

The use of ester and DTC derivatized calixarene in the functionalization of metal NPs induces the adsorption of molecules that cannot interact with the metal surface and, then, cannot render a SERS spectrum. Examples of these molecules are the PAHs, which do not have the possibility of interacting with the metal due to the absence of any functional group in their structure. DCEC and DTCX have been successfully employed in the selective and sensitive detection of PAHs on Ag NPs $[54,56]$.

\section{Conclusions}

The metal-adsorbate interaction is a crucial issue to understanding why some adsorbates give rise to intense SERS spectra while others are practically inactive. The interaction with the metal is a way to ensure the approach of the target molecule to the surface. Only when a molecule is in close contact with the metal surface can it undergo a marked intensification by means of the two main mechanisms described to account for the giant Raman scattering intensification observed on nanostructured metal surfaces. From the analysis of the SERS and SEIRA spectra, one can deduce the interaction mechanism of the adsorbed species.

In wet systems, the presence of $\mathrm{O}, \mathrm{N}$, or $\mathrm{S}$ atoms is normally needed for a strong interaction to take place. This interaction occurs through the formation of CT complexes, the adsorbate acting as a donor ligand and the metal as electron acceptor. Indeed, this $\mathrm{CT}$ is necessary to establish a coordination complex between the adsorbate and the metal and should not be confused with the CT invoked by the mechanism of the same name to account for the enhancement of Raman signal on NPs.

In some cases, the interaction is so strong that a chemical change is induced in the adsorbates. These changes can proceed through bond cleavages or through polymerization. A particularly strong interaction was detected in the case of bypyridinium cations and DTC-containing molecules.

In addition, electrostatic forces may play an important role in the interaction with the NPs' metal surface. Positively charged adsorbates are good candidates to be readily adsorbed on the negatively charged surface of metal NPs. In this case, the interaction occurs through anionic species adsorbed on the metal through ionic pair formation also involving anionic species previously adsorbed onto the surface: citrate, nitrate, chloride, etc.

The interfacial properties of metal surfaces can be modified to permit the adsorption of molecular species or to hinder the interaction of other nondesired adsorbates [57]. The presence of halides induces the adsorption of ammonium species through the formation of ionic pairs and avoids the adsorption of carboxylate species. The presence of halides also passivates the surface, avoiding the chemical degradation undergone by O-containing species.

Surface functionalization with bypyridinium cations and calixarene compounds acting as hosts can be applied in the 
detection of nonactive SERS molecules, such as polycyclic aromatic hydrocarbons. Thus, the main conclusion drawn from this paper is that the knowledge derived from the study of the interaction occurring between metals and adsorbates is very useful to increase the sensitivity of NPbased systems and, thus, in the design of advanced surface systems applicable in sensor devices.

Acknowledgements The authors acknowledge projects FIS200400108 from the Spanish Ministerio de Educación y Ciencia, MICROSERES S-0505/TIC-0191 from Comunidad de Madrid, Fondecyt 1040640 and 1070078 from Conicyt, and C-13879 from Fundación Andes. L. G. acknowledges an I3P fellowship from Consejo Superior de Investigaciones Cientificas and Z. J. acknowledges the European Community's Sixth Framework Programme for Marie Curie Early Stage Research Training Fellowship (contract number MEST-CT2004-513915.)

\section{References}

1. Aroca R (2006) Surface-enhanced vibrational spectroscopy. Wiley, Chichester

2. Knoll W, Fritzsche W (2005) Nanoparticles for biotechnology applications. IEE Proc Nanobiotechnol 152:1

3. Popov AK, Brummer J, Tanke RS, Taft G, Loth M, Langlois R, Wruck A, Schmitz R (2006) Synthesis of isolated silver nanoparticles and their aggregates manipulated by light. Laser Phys Lett 11:546-552

4. Moskovits M (2005) Surface-enhanced Raman spectroscopy: a brief retrospective. J Raman Spectrosc 36:485-496

5. Burstein E, Chen YJ, Chen CY, Lundquist S, Tosatti E (1979) Giant Raman-scattering by adsorbed molecules on metal surfaces. Solid State Commun 29:567-570

6. Kovacs GJ, Loutfy RO, Vincent PS, Jennings C, Aroca R (1986) Distance dependence of SERS enhancement factor from Langmuir-Blodgett monolayers on metal island films - Evidence for the electromagnetic mechanism. Langmuir 2:689-694

7. Otto A, Mrozek I, Grabhorn H, Akemann W (1992) Surfaceenhanced Raman-scattering. J Phys Condens Matter 4:1143-1212

8. Houk KN, Leach AG, Kim SP, Zhang X (2003) Binding affinities of host-guest, protein-ligand, and protein-transition-state complexes. Angew Chem Int Ed Engl 42:4872-4897

9. Cañamares MV, García-Ramos JV, Gómez-Varga D, Domingo C, Sanchez-Cortes S (2005) Comparative study of the morphology, aggregation, adherence to glass, and surface-enhanced Raman scattering activity of silver nanoparticles prepared by chemical reduction of $\mathrm{Ag}+$ using citrate and hydroxylamine. Langmuir 21:8546-8553

10. Garrell RL, Shaw KD, Krim S (1983) Surface enhanced Ramanspectroscopy of halide-ions on colloidal silver - Morphology and coverage dependence. Surf Sci 124:613-624

11. Shipway AN, Katz E, Willner I (2000) Nanoparticle arrays on surfaces for electronic, optical, and sensor applications. Chem Phys Chem 1:18-52

12. Gao P, Weaver MJ (1986) Metal adsorbate vibrational frequencies as a probe of surface bonding - Halides and pseudohalides at gold electrodes. J Phys Chem 90:4057-4063

13. Moskovits M, Suh JS (1984) Surface selection-rules for Surfaceenhanced Raman-spectroscopy - Calculations and application to the surface-enhanced Raman-spectrum of phthalazine on silver. J Phys Chem 88:5526-5530
14. Gao XP, Davies JP, Weaver MJ (1990) A test of surface selectionrules for surface-enhanced Raman-scattering - The orientation of adsorbed benzene and monosubstituted benzenes on gold. J Phys Chem 94:6858-6864

15. Carter DA, Pemberteon JE (1992) Surface-enhanced Ramanscattering of the acid-base forms of imidazole on Ag. Langmuir 8:1218-1225

16. Aroca RF, Alvarez-Puebla RA, Pieczonka N, Sanchez-Cortes S, Garcia-Ramos JV (2005) Surface-enhanced Raman scattering on colloidal nanostructures. Adv Colloid Interface Sci 116:45-61

17. Sanchez-Cortes S (2004) SERS on colloids. Opt Pur Apl 37:35-42

18. Cañamares MV, García-Ramos JV, Gómez-Varga D, Domingo C, Sanchez-Cortes S (2007) Ag nanoparticles prepared by laser photoreduction as substrates for in situ surface-enhanced Raman scattering analysis of dyes. Langmuir 23:5210-5215

19. Domingo C, Resta V, Sanchez-Cortes S, García-Ramos JV, Gonzalo J (2007) Pulsed laser deposited Au nanoparticles as substrates for surface-enhanced vibrational spectroscopy. J Phys Chem C 111:8149-8152

20. Gui J, Devine TM (1989) Influence of hydroxide on the SERS of water and chloride. Surf Sci 224:525-542

21. Nichols RJ, Burgess I, Young KL, Zamlynny V, Lipkowski J (2004) A quantitative evaluation of the adsorption of citrate on $\mathrm{Au}$ (111) using SNIFTIRS. J Electroanal Chem 563:33-39

22. Sanchez-Cortes S, Francioso O, García-Ramos JV, Ciavatta C, Gessa C (2001) Catechol polymerization in the presence of silver surface. Coll Surf A 176:177-184

23. Sanchez-Cortes S, García-Ramos JV (2000) FT surface-enhanced Raman evidence of the oxidative condensation reactions of caffeic acid in solution and on silver surface. Appl Spectrosc 54:230-238

24. Alvarez-Ros M, Sanchez-Cortes S, Francioso O, García-Ramos JV (2001) Catalytic modification of gallic acid on a silver surface studied by surface-enhanced Raman spectroscopy. J Raman Spectrosc 32:143-145

25. Fabriciova G, García-Ramos JV, Miskovsky P, Sanchez-Cortes S (2002) Adsorption mechanism and acidic behavior of naphthazarin on Ag nanoparticles studied by Raman spectroscopy. Vib Spectrosc 30:203-212

26. Cañamares MV, García-Ramos JV, Domingo C, Sanchez-Cortes S (2004) Surface-enhanced Raman scattering study of the adsorption of the anthraquinone pigment alizarin on $\mathrm{Ag}$ nanoparticles. J Raman Spectrosc 35:921-927

27. Jurasekova Z, García-Ramos JV, Domingo C, Sanchez-Cortes S (2006) Surface-enhanced Raman scattering of flavonoids. J Raman Spectrosc 37:1239-1241

28. Minero C, Mariella G, Maurino V, Vione D, Pelizzetti E (2000) Photocatalytic transformation of organic compounds in the presence of inorganic ions. 2. Competitive reactions of phenol and alcohols an a titanium dioxide-fluoride system. Langmuir 16:8964-8972

29. Fleischmann FM, Hendra PJ, McQuillan AJ (1974) Ramanspectra of pyridine adsorbed at a silver electrode. Chem Phys Lett 26:163-166

30. Muniz-Miranda M (1996) Surface enhanced Raman scattering of 4,4-bipyridine adsorbed on smooth copper, silver and aluminium surfaces activated by deposited silver particles. J Raman Spectrosc 27:435-437

31. Arenas JF, Woolley MS, Otero JC, Marcos JI (1996) Chargetransfer processes in surface-enhanced Raman scattering. FranckCondon active vibrations of pyrazine. J Phys Chem 100: 3199-3206

32. Herne TM, Ahern AM, Garrell RL (1991) Surface-enhanced Raman-spectroscopy of peptides - Preferential n-terminal adsorption on colloidal silver. J Am Chem Soc 113:846-854

33. Castro JL, López-Ramírez MR, López-Tocón I, Otero JC (2003) Vibrational study of the metal-adsorbate interaction of phenylacetic 
acid and alpha-phenylglycine on silver surfaces. J Colloid Interface Sci 263:357-363

34. Oh ST, Kim K, Kim MS (1991) Adsorption and surface-reaction of acridine in silver sol - Surface-enhanced Raman-spectroscopic study. J Phys Chem 95:8844-8849

35. Levi G, Pantigny J, Marsault JP, Aubard J (1993) SER spectra of acridine and acridinium ions in colloidal silver sols - Electrolytes and ph effects. J Raman Spectrosc 24:745-752

36. Murza A, Sanchez-Cortes S, García-Ramos JV (1998) Fluorescence and surface-enhanced Raman study of 9-aminoacridine in relation to its aggregation and excimer emission in aqueous solution and on silver surface. Biospectroscopy 4:327-339

37. Sanchez-Cortes S, García-Ramos JV (1992) SERS of cytosine and its methylated derivatives on metal colloids. J Raman Spectrosc 23:61-66

38. Camafeita LE, Sanchez-Cortes S, García-Ramos JV (1995) SERS of cytosine and its methylated derivatives on gold sols. J Raman Spectrosc 26:149-154

39. Sanchez-Cortes S, García-Ramos JV (2001) Influence of coverage in the surface-enhanced Raman scattering of cytosine and its methyl derivatives on metal colloids: chloride and $\mathrm{pH}$ effects. Surf Sci 473:133-142

40. Feng Q, Cotton TM (1986) Surface-enhanced resonance Ramanstudy of the photoreduction of methylviologen on a p-inp semiconductor electrode. J Phys Chem 90:983-987

41. Millán JI, Garcia-Ramos JV, Sanchez-Cortes S, Rodríguez-Amaro R (2003) Adsorption of lucigenin on Ag nanoparticles studied by surface-enhanced Raman spectroscopy: effect of different anions on the intensification of Raman spectra. J Raman Spectrosc 34:227-233

42. Millán JI, Garcia-Ramos JV, Sanchez-Cortes S (2003) Study of the adsorption and electrochemical reduction of lucigenin on $\mathrm{Ag}$ electrodes by surface-enhanced Raman spectroscopy. J Electroanal Chem 556:83-92

43. Carrasco EA, Campos-Vallette FM, Leyton P, Diaz GF, Clavijo RE, García-Ramos JV, Inostroza N, Domingo C, Sanchez-Cortes S, Koch R (2003) Study of the interaction of pollutant nitro polycyclic aromatic hydrocarbons with different metallic surfaces by surface-enhanced vibrational spectroscopy (SERS and SEIR). J Phys Chem 107:9611-9619

44. Domingo C, García-Ramos JV, Sanchez-Cortes S, Aznarez JA (2004) Surface-enhanced infrared absorption of DMIP on goldgermanium substrates coated by self-assembled monolayers. J Mol Struct 661:419-427

45. Seelenbinder JA, Brown CW (2002) Comparison of organic selfassembled monolayers as modified substrates for surface- enhanced infrared absorption spectroscopy. Appl Spectrosc 56:295-299

46. Sanchez-Cortes S, Vasina M, Francioso O, García-Ramos JV (1998) Raman and surface-enhanced Raman spectroscopy of dithiocarbamate fungicides. Vib Spectrosc 17:133-144

47. Sanchez-Cortes S, Domingo C, García-Ramos JV, Aznarez JA (2001) Surface-enhanced vibrational study (SEIR and SERS) of dithiocarbamate pesticides on gold films. Langmuir 17:1157-1162

48. Sanchez-Cortes S, Molina M, García-Ramos JV, Carmona P (1991) Interactions of cytidine derivatives with metals as revealed by surface-enhanced Raman-spectroscopy. J Raman Spectrosc 22:819-824

49. Rivas L, Sanchez-Cortes S, García-Ramos JV (2002) Conformational study of AZT in aqueous solution and adsorbed on a silver surface by means of Raman spectroscopy. J Raman Spectrosc 33:6-9

50. Dijkstra S, Benevides J, Thomas GJ Jr (1991) Raman spectral studies of nucleic-acids .40. Solution conformations of nucleoside analogs exhibiting antiviral activity against human-immunodeficiency-virus. J Mol Struct 242:283-301

51. He J, Zhou H, Wan F, Lu Y, Xue G (2005) SERS study of the high quality conducting polythiophene film. Vib Spectrosc 31:265-269

52. Martin F, Prieto AC, De Saja JA, Aroca R (1998) SERS study of the pyrrole polymerization. J Mol Struct 174:363-368

53. Pérez-Méndez M, Marsal-Berenguel R, Sanchez-Cortes S (2004) Adsorption of a cholesteric liquid crystal polyester on silver nanoparticles studied by surface enhanced Raman scattering and micro Raman spectroscopy. Appl Spectrosc 58:562-569

54. Leyton P, Sanchez-Cortes S, García-Ramos JV, Domingo C, Campos-Vallette M, Saitz C, Clavijo RE (2004) Selective molecular recognition of polycyclic aromatic hydrocarbons (PAHs) on calix[4]arene-functionalized Ag nanoparticles by surfaceenhanced Raman scattering. J Phys Chem B 108:17484-17490

55. Leyton P, Domingo C, Sanchez-Cortes S, Campos-Vallette M, Diaz GF, García-Ramos JV (2007) Reflection-absorption IR and surface-enhanced IR spectroscopy of tetracarboethoxy t-butylcalix[4]arene, as a host molecule with potential applications in sensor devices. Vib Spectrosc 43:358-365

56. Guerrini L, García-Ramos JV, Domingo C, Sanchez-Cortes S (2006) Functionalization of $\mathrm{Ag}$ nanoparticles with dithiocarbamate calix[4]arene as an effective supramolecular host for the surface-enhanced Raman scattering detection of polycyclic aromatic hydrocarbons. Langmuir 22:10924-10926

57. Sanchez-Cortes S, García-Ramos JV (1998) Anomalous Raman bands appearing in surface-enhanced Raman spectra. J Raman Spectrosc 29:365-371 